Erich Jonasch - Unfallchirurgische Operationen 



\title{
UNFALLCHIRURGISCHE OPERATIONEN
}

\author{
INDIKATION - TECHNIK - FEHLER \\ VON \\ DR. MED. ERICH JONASCH \\ FACHARZT FOR UNFALLCHIRURGIE \\ OBERARZT AM UNFALLKRANKENHAUS WIEN XX DER ALLGEMEINEN \\ UNFALLVERSICHER UNGSANSTALT
}

GELEITWORT VON PROFESSOR DR. MED. LORENZ BƠHLER

112 ABBILDUNGEN IN 301 EINZELDARSTELLUNGEN

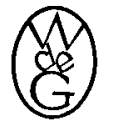

WALTER DE GRUYTER \& CO.

VORMALS G. J. GOSCHEN'SCHE VERLAGSHANDLUNG · J. GUTTENTAG

VERLAGSBUCHHANDLUNG . GEORG REIMER - KARL J.TRUBNER VEIT \& COMP.

BERLIN 1965 
Copyright 1965 by Walter de Gruyter \& Co., vormals G. J. Göschen'sche Verlagshandlung, J. Guttentag, Verlagsbudhhandlung Georg Reimer, Karl J. Trübner, Veit \& Comp., Berlin 30, Genthiner Straße 13 - Alle Rechte, auch die des auszugsweisen Nachdrucks, der photomechanischen Wiedergabe, der Herstellung von Mikrofilmen und der Ubesetzung, vorbehalten Archiv-Nr. 5615651 - Printed in Germany - Satz und Drudk: Buchdrudkerei Franz Spiller, 1 Berlin 36. 